\title{
Cinética de fermentação ruminal in vitro de dietas com inclusão do resíduo de feiião (Phaseolus vulgaris L.) substituindo a torła de algodão
}

\author{
Ferro, M.M. ${ }^{1} ;$; Zanine, A.M. ${ }^{2}$, Ferro, R.M. ${ }^{3}$ e Souza, A.L. ${ }^{4}$
}

\author{
'Universidade Federal de Mato Grosso. Campus Cuiabá. Cuiabá. MT. Brasil. \\ ${ }^{2}$ Universidade Federal do Maranhão. Campus Chapadinha. Chapadinha. MA. Brasil \\ ${ }^{3}$ Universidade Federal de Mato Grosso. Campus Sinop. Sinop. MT. Brasil. \\ ${ }^{4}$ Universidade Federal de Mato Grosso. Campus Rondonópolis. Rondonópolis. MT. Brasil
}

\section{PalAVRAS-ChaVE ADICIONAIS}

Degradação.

Latência.

Parâmetros.

Produção de gás.

\section{RESUMO}

Objetivou-se com esta pesquisa avaliar os parâmetros fermentativos ruminal in vitro da inclusão de resíduo de feijão (Phaseolus vulgaris L.) em substituição à torta de algodão. Os tratamentos experimentais foram constituídos pela substituição da torta de algodão nos níveis de 0, 33, 66 e 100\% por resíduo de feijão. O preparo das amostras foi realizado utilizando 500 mg de amostra seca ao ar, moída a $1 \mathrm{~mm}$, onde imediatamente após a adição do inoculo, os frascos receberam tampa de borracha, lacre de alumínio e foram colocados em banho-maria, com agitação orbital, a $39^{\circ} \mathrm{C}$. As leituras de pressão foram realizadas por meio de um transdutor de pressão nos seguintes tempos: $1,2,4,6,8,10,12,15,18,24,30,36,48,60,72,84$ e 96 horas. A cinética da produção cumulativa dos gases foi analisada empregando-se o modelo logístico bicompartimental. O volume final de gases da fração de carboidratos não-fibrosos das dietas não apresentou efeito significativo $(p>0,05)$, enquanto a taxa de degradação da fração de carboidratos não-fibrosos, o volume de gases da fração de carboidratos fibrosos, a taxa de degradação da fração de carboidratos não-fibrosos e a latência apresentaram aumento linear significativo $(p<0,05)$. A adição do resíduo de feiião em substituição à torta de algodão potencializa uma maior disponibilização de nutrientes devido à melhora dos parâmetros ruminais in vitro.

\section{Kinetics in vitro ruminal fermentation of diets with inclusion of bean (Phaseolus} vulgaris $L$.) residue replacing cottonseed meal

\section{SUMMARY}

The objective of this research was to evaluate the ruminal fermentation parameters of in vitro bean (Phaseolus vulgaris L.) residue inclusion in replacement of cottonseed meal. The experimental treatments were the replacement of cottonseed meal at levels of $0,33,66$ and $100 \%$ by bean residue. The sample preparation was performed using $500 \mathrm{mg}$ of air dried sample, ground to $1 \mathrm{~mm}$, inwhich, right after the addition of the inoculum, flasks received rubber stopper, aluminum seal and were placed in a water bath, with orbital shaking, at $39^{\circ} \mathrm{C}$. Pressure readings were taken using a pressure transducer at the following times: 1,2 , $4,6,8,10,12,15,18,24,30,36,48,60,72,84$ and 96 hours. The kinetics of cumulative production of gas was analyzed using a logistic two-compartment model. The final gas volume fraction of non-fiber carbohydrate diets had no significant effect $(p>0.05)$, while the rate of degradation of the fraction of non-fibrous carbohydrates, the volume fraction of fiber carbohydrates gases, the degradation rate of the fraction of non-fibrous carbohydrates and latency showed a significant linear increase $(\mathrm{p}<0.05)$. The addition of the bean residue to replace cotton cake potentiates a greater availability of nutrients because of an improved in vitro ruminal fermentation.

\section{INFORMATION}

\section{Cronología del artículo.}

Recibido/Received: 30.11 .2015

Aceptado/Accepted: 08.02.2017

On-line: 15.07.2017

Correspondencia a los autores/Contact e-mail:

mmf_zootecnia@yahoo.com.br

\section{INTRODUÇÃO}

Diversos trabalhos vêm sendo desenvolvidos com a inclusão de subprodutos agroindustriais em dietas de ruminantes, e os resultados demonstram que existem grandes possibilidades de aproveitamento destes alimentos na alimentação animal, podendo ser utilizados 
como ingredientes de dietas, em associação com outros alimentos (Rodrigues Filho et al., 2001).

O resíduo de feijão (Phaseolus vulgaris L.) é um alimento proveniente do beneficiamento da agroindústria, que apresenta disponibilidade em diversos estados brasileiros, com uma produção média de 3 mil toneladas (CONAB, 2014), permitindo sua inclusão em dietas de animais ruminantes em até 15\% (Ferro, 2014; Fonseca, 2015).

O resíduo de feijão pode ser classificado como alimento concentrado protéico, devido seu elevado teor de proteína bruta. Apresenta em média uma composição de $89 \%$ matéria seca (MS), 23\% proteína bruta (PB), 21\% de fibra em detergente neutro (FDN), 9\% de fibra em detergente ácido (FDA), 7,81\% de proteína insolúvel em detergente neutro (PIDN), 1,51\% de proteína insolúvel em detergente ácido (PIDA), 1,67\% de extrato etéreo (EE), 5,11\% de matéria mineral (MM), 1,4\% de lignina, 72,61\% de carboidratos totais (CT) e $34,9 \%$ de carboidratos não-fibrosos (CNF) (Magalhães et al., 2008; Marcondes et al., 2009; Azevêdo et al., 2011).

O resíduo de feijão é um produto resultante do beneficiamento do feijão destinado ao consumo humano, constituído de grãos avariados, inclusive os de outras variedades e ou classes que não a predominante, em mistura com impurezas (MAPA, 2002).

Desta forma, para melhor aproveitamento dos subprodutos e resíduos é de fundamental importância o conhecimento das propriedades físicas e químicas desses alimentos, uma vez que, eles possuem características distintas de degradação e passagem pelo trato gastrintestinal, quando comparados com as forragens (Armentano e Pereira, 1997). Estas peculiaridades podem afetar o consumo, que é influenciado, entre outros fatores, pela estrutura física e composição química das dietas (Carvalho et al., 2004), representando o principal fator determinante do ganho de peso (Piona, 2010).

Por conseguinte, a estimação das taxas de degradação dos constituintes dos alimentos é fundamental para adequação dietética e predição do desempenho (Cabral et al., 2004). O método que mensura a produção cumulativa dos gases pode ser utilizado para estimar as taxas de degradação das frações solúveis e insolúveis dos carboidratos, oriundos do metabolismo microbiano (Pell e Schofield, 1993; Malafaia et al., 1998).

Assim, objetivou-se com esta pesquisa avaliar os parâmetros fermentativos ruminal in vitro com a inclusão de resíduo de feijão em substituição à torta de algodão.

\section{MATERIAL E MÉTODOS}

As incubações foram realizadas no laboratório de Nutrição Animal da Universidade Federal de Mato Grosso - Campus Cuiabá-MT.

As dietas experimentais foram compostas com $45 \%$ de volumoso, utilizando como fonte silagem de capim elefante (Pennisetum purpureum 'Napier') e 55\% de concentrado, na matéria seca, sendo as mesmas isoprotéicas com 14\% de PB (NRC, 2007). Os tratamentos experimentais foram constituídos pela substituição da torta de algodão nos níveis de 0,33, 66 e 100\% por resíduo de feijão no concentrado, correspondendo a inclusão de 0 , 8,16 e $24 \%$ na dieta. O resíduo de beneficiamento de feijão foi constituído de grãos avariados dos tipos: inteiros (amassados, enrugados, manchados e despeliculados), partidos (bandinhas sadias) ou quebrados (pedaços sadios), além de impurezas, como talos e vagens.

As amostras dos alimentos volumosos foram présecos em estufa de ventilação forçada sob $55^{\circ} \mathrm{C}$ durante 72 horas para determinação da matéria seca (MS). Em seguida, todos os alimentos foram moídos em peneira de $1 \mathrm{~mm}$ para posteriormente serem analisados para proteína bruta $(\mathrm{PB})$, matéria mineral $(\mathrm{MM})$, extrato etéreo (EE), fibra detergente neutro (FDN) e fibra detergente ácido (FDA), conforme metodologias descritas por Detmann et al. (2012). O resíduo da fibra em detergente neutro corrigido para cinzas e compostos nitrogenados (FDNcp) foi determinado conforme Licitra et al. (1996).

Os carboidratos totais (CT) foram calculados a partir da equação: CT $=100-(\% \mathrm{~PB}+\% \mathrm{MM}+\% \mathrm{EE})$, de acordo com Sniffen et al. (1992). A fibra em detergente neutro indigestível (FDNi) dos alimentos foi obtida através da incubação in situ por 240 horas (Casali et al., 2008), seguida da análise de fibra em detergente neutro. A composição química dos alimentos e das dietas estão sendo apresentadas na tabela I e tabela II, respectivamente.

O preparo das amostras foi realizado utilizando $500 \mathrm{mg}$ de amostra seca ao ar moída a $1 \mathrm{~mm}$, conforme Pell e Schofield (1993). Em seguida as amostras foram alocadas em frascos de vidro cor âmbar com capacidade de $120 \mathrm{~mL}$, utilizando-se 12 frascos por tratamento, totalizando 48 frascos.

Aos frascos foram adicionados $40 \mathrm{~mL}$ de solução tampão de McDougall (McDougall, 1948), previamente reduzido com $\mathrm{CO}_{2}\left(\mathrm{pH} \mathrm{6,9-7,0),} \mathrm{mantidos} \mathrm{a} 39^{\circ} \mathrm{C}\right.$. Meia hora após, foram adicionados $10 \mathrm{~mL}$ de inoculo ruminal sob aspersão de $\mathrm{CO}_{2}$. O líquido ruminal foi proveniente de animais previamente adaptados as dietas experimentais. Após ser coado em peneira, o líquido ruminal foi acondicionado em garrafa térmica e levado para o laboratório, sendo toda a sua manipulação realizada sob aspersão de $\mathrm{CO}_{2}$, livre de oxigênio. Imediatamente após a adição do inoculo, os frascos receberam tampa de borracha, lacre de alumínio e colocados em banho-maria, com agitação orbital, a $39^{\circ} \mathrm{C}$. As leituras de pressão (psi - pressão por polegada quadrada) foram realizadas por meio de um transdutor de pressão (Datalogger Pressure ${ }^{\circledR}$ ) semi-automático, nos seguintes tempos: 1, 2, 4, 6, 8, 10, 12, 15, 18, 24, 30, 36, 48, 60, 72, 84 e 96 horas.

Para descontar o volume de gases oriundo do líquido de rúmen e da solução tampão, dois frascos foram incubados sem amostra (branco). Dessa forma, para cada tempo de leitura, o volume de gases dos frascos com amostra foi subtraído do volume dos frascos sem amostra.

Com o somatório do volume de gases para cada tempo de leitura, foram estabelecidas as curvas de produção cumulativa dos gases. A conversão de psi para $\mathrm{mL}$ foi feita a partir da equação de regressão linear 


\begin{tabular}{|c|c|c|c|c|c|}
\hline \multirow{2}{*}{ Componentes } & \multicolumn{5}{|c|}{ Alimentos ${ }^{4}(\mathrm{~g} / \mathrm{kg} \mathrm{MS})$} \\
\hline & SCN & FS & MI & TA & RF \\
\hline Matéria seca & 220,00 & 886,70 & 921,00 & 935,90 & 899,40 \\
\hline Matéria orgânica & 891,90 & 933,10 & 988,60 & 954,10 & 958,10 \\
\hline Proteína bruta & 63,10 & 538,10 & 84,30 & 306,90 & 207,00 \\
\hline Extrato etéreo & 28,70 & 21,00 & 54,00 & 144,00 & 24,90 \\
\hline Matéria mineral & 108,00 & 66,80 & 11,30 & 45,80 & 41,80 \\
\hline FDNcp ${ }^{1}$ & 583,40 & 224,80 & 218,20 & 352,10 & 190,90 \\
\hline Fibra em detergente ácido & 495,50 & 198,70 & 54,00 & 353,40 & 138,20 \\
\hline Carboidratos não-fibrosos & 216,80 & 149,30 & 632,20 & 151,20 & 535,40 \\
\hline FDNi & 250,00 & 15,70 & 7,50 & 102,00 & 13,90 \\
\hline PIDN & 245,00 & 383,30 & 383,30 & 183,20 & 171,60 \\
\hline $\mathrm{NDT}_{\text {est }}$ & 503,00 & 734,20 & 846,90 & 613,70 & 781,30 \\
\hline
\end{tabular}

MS: Matéria seca; FDNcp: fibra detergente neutro corrigido para cinzas e proteína; FDNi: fibra em detergente neutro indigestível; PIDN

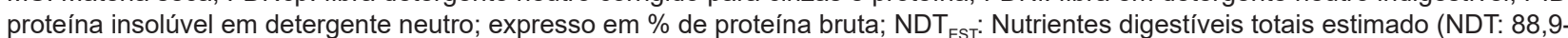
$(0,779 * \%$ de fibra em detergente ácido) (Paterson, 2000); SCN: silagem de capim napier; FS: farelo de soja; MI: milho moído; TA: torta de algodão; RF: resíduo de feijão.

$(Y=a+b x)$ em que o coeficiente " $b$ " da equação possibilitou a correção e transformação de pressão (psi) em volume de gases $(\mathrm{mL})$ corrigido para a pressão barométrica do dia. Para isso, injetou-se volume conhecido de gases em frascos mantidos sob as mesmas condições das amostras incubadas.

A cinética da produção cumulativa dos gases foi analisada empregando-se o modelo logístico bicompar-

Tabela II. Composição centesimal e química das dietas experimentais, expressos com base na matéria seca (MS) (Chemical composition of experimental diets expressed basing on dry matter (MS)).

\begin{tabular}{|c|c|c|c|c|}
\hline \multirow{3}{*}{ Componentes } & \multicolumn{4}{|c|}{ Níveis de substituição da torta de algodão por resíduo de feijão (\%) } \\
\hline & 0 & 33 & 66 & 100 \\
\hline & \multicolumn{4}{|c|}{ Composição centesimal (g/kg de MS) } \\
\hline $\begin{array}{l}\text { Silagem de capim } \\
\text { elefante }\end{array}$ & 450,0 & 450,0 & 450,0 & 450,0 \\
\hline Milho moído & 280,0 & 274,0 & 273,0 & 274,0 \\
\hline Farelo de soja & 28,0 & 3,0 & 3,0 & 3,0 \\
\hline Torta de algodão & 240,0 & 160,0 & 8,0 & 0,0 \\
\hline Resíduo de feijão & 0,0 & 80,0 & 160,0 & 239,0 \\
\hline \multirow[t]{2}{*}{ Ureia } & 0,0 & 2,0 & 3,0 & 4,0 \\
\hline & \multicolumn{4}{|c|}{ Composição química (g/kg de MS) } \\
\hline Matéria seca & 590,20 & 587,20 & 584,40 & 581,50 \\
\hline Matéria orgânica & 935,20 & 933,10 & 932,30 & 932,00 \\
\hline Proteína bruta & 141,30 & 141,40 & 136,20 & 130,00 \\
\hline Extrato etéreo & 63,30 & 53,50 & 43,90 & 34,40 \\
\hline Matéria mineral & 64,70 & 64,60 & 64,20 & 63,90 \\
\hline FDNcp ${ }^{1}$ & 414,90 & 401,60 & 388,40 & 375,40 \\
\hline $\begin{array}{l}\text { Fibra em detergente } \\
\text { ácido }\end{array}$ & 328,80 & 312,10 & 294,70 & 277,30 \\
\hline $\begin{array}{l}\text { Carboidratos não- } \\
\text { fibrosos }\end{array}$ & 315,70 & 342,90 & 373,50 & 403,70 \\
\hline $\mathrm{FDNi}^{1}$ & 139,60 & 132,60 & 125,50 & 118,40 \\
\hline PIDN $^{1,2}$ & 272,90 & 271,10 & 269,80 & 268,90 \\
\hline $\mathrm{NDT}_{\text {est }}{ }^{3}$ & 632,80 & 643,90 & 656,40 & 669,40 \\
\hline
\end{tabular}

${ }^{1}$ FDNcp: fibra detergente neutro corrigido para cinzas e proteína; FDNi: fibra em detergente neutro indigestível; PIDN: proteína insolúvel em detergente neutro; ${ }^{2}$ expresso em \% de proteína bruta; NTD ${ }_{\text {est }}$ Nutrientes digestíveis totais estimado (NDT: $88,9-\left(0,779^{*} \%\right.$ de fibra em detergente ácido) (Paterson, 2000). 
timental de Schofield et al. (1994), onde: $\mathrm{V}(\mathrm{t})=\mathrm{Vf}_{1} /(1+$ $\exp \left(2-4^{*} \mathrm{C}_{1}^{*}(\mathrm{~T}-\mathrm{L})\right)+\mathrm{Vf}_{2} /\left(1+\exp \left(2-4^{*} \mathrm{C}_{2}{ }^{*}(\mathrm{~T}-\mathrm{L})\right)\right.$, no qual $\mathrm{V}(\mathrm{t})$ é o volume de gases acumulado no tempo $\mathrm{t} ; \mathrm{Vf}_{1}$, o volume de gases oriundo da fração de rápida digestão; $\mathrm{c}_{1}\left(\mathrm{~h}^{-1}\right)$, a taxa de degradação da fração de rápida digestão; $\mathrm{T}$, o tempo (h); L, a latência; $\mathrm{Vf}_{2}$, o volume de gases da fração de lenta digestão; $c_{2}\left(h^{-1}\right)$, a taxa de degradação da fração de lenta digestão.

A energia metabolizável (EM, MJ/kg MS) e digestibilidade in vitro da matéria orgânica (DMO, g/kg MO) foram estimadas de acordo com Menke et al. (1979) como:

$\mathrm{EM}=2,20+0,136 \mathrm{PG}(\mathrm{mL} / 0,200 \mathrm{~g} \mathrm{MS})+0,057 \mathrm{~PB}$ (mg/0,200 g MS)

$\mathrm{DMO}=148,8+8,89 \mathrm{PG}+4,5 \mathrm{~PB}(\mathrm{mg} / 0,200 \mathrm{~g} \mathrm{MS})+$ 0,651 cinzas (mg/0,2 g MS)

Sendo que PG é a produção de gás em mL de 200 $\mathrm{mg}$ de amostra seca incubada por $24 \mathrm{~h}$.

A concentração de ácidos graxos de cadeia curta (AGCC) são calculados de acordo com Getachew et al. (2002):

\section{AGCC (mmol/g MS) = 0,0222PG-0,00425}

As variáveis foram analisadas através da análise de variância, e quando significativo a 5\% de probabilidade, fez analise de regressão utilizando o PROC MIXED do SAS 9.2.

\section{RESULTADOS E DISCUSSÃO}

A produção cumulativa de gases variou de 47,47 a 150,61 mL/g MS para torta de algodão e milho grão, respectivamente (tabela III). Nota-se que a maior produção cumulativa de gases foi para milho grão, seguido do resíduo de feijão, farelo de soja e da silagem de capim elefante, sendo o menor valor estimado para a torta de algodão (figura 1). Estes dados permitem inferir a elevada sensibilidade da técnica de produção cumulativa de gases à variação na disponibilidade de nutrientes nos alimentos, notadamente carboidratos não-fibrosos, presentes em maior concentração no milho e resíduo de feijão (tabela I).

A taxa de degradação da fração carboidratos nãofibrosos (KdCNF) apresentou-se superior para a torta de algodão, seguido do milho e farelo de soja, sendo o resíduo de feijão e a silagem de capim napier os alimentos que apresentaram menores taxas de degradação (tabela III). O volume de gases da fração carboidratos fibrosos (VFCHOF) foi maior para o milho, seguido do resíduo de feijão, farelo de soja, silagem de capim napier e torta de algodão (tabela III). A taxa de degradação da fração carboidratos fibrosos (KdCHOF) foi superior para milho, farelo de soja e torta de algodão, seguido de resíduo de feijão e silagem de capim napier (tabela III).

O tempo de latência foi menor para silagem de capim napier, seguido farelo de soja, torta de algodão, resíduo de feijão e milho.

Marcondes et al. (2009) avaliando a degradação potencial de alguns alimentos encontrou resultados contrários ao presente estudo, obtendo taxas de degradação maiores para farelo de soja e resíduo de feijão e menores para farelo de algodão, resíduo este obtido pelo beneficiamento do algodão. Acredita-se que esta diferença esteja relacionada a composição do alimento, uma vez que o resíduo de feijão utilizado no presente estudo apresentava-se com uma quantidade de impurezas superior, podendo ter influenciado na sua composição, uma vez que o seu teor de FDNcp foi de $19 \%$ enquanto o resíduo utilizado por Marcondes et al. (2009) apresentava 14\%.

Acredita-se que outro fator que pode ter interferido na disponibilidade dos nutrientes do resíduo de feijão pode estar relacionado a alguns fatores antinutricionais (Sgarbieri (1996) citado por Magalhães (2005)) que podem levar a uma menor disponibilidade de proteína, prejudicando desta forma os parâmetros, uma vez que, estamos trabalhando com alimentos que sua composição apresenta alta concentração de proteínas.

Corroborando com os resultados acima discutidos, observa-se na figura 2 que o perfil de fermentação das dietas foram muito parecidos até 12 horas de incubação, sobressaindo-se à dieta com $100 \%$ de substituição da torta de algodão por resíduo de feijão a partir das 15 horas de incubação, seguida da dieta com 33 e $66 \%$ de substituição, sendo a dieta com $0 \%$ de substituição a que apresentou menor produção cumulativa de gases a partir da 15 horas de incubação.

O volume final de gases da fração CNF (VFCNF) das dietas não apresentou efeito significativo ( $p>0,05)$ com o aumentou dos níveis de substituição da torta de algodão por resíduo de feijão apresentando uma média 17,24 mL/g de MS (tabela IV). Esperava-se aumento

Tabela III. Parâmetros cinéticos de fermentação ruminal in vitro pela técnica cumulativa de gases dos alimentos (Kinetic parameters of in vitro ruminal fermentation by cumulative technique of food gases).

\begin{tabular}{lccccc}
\hline Alimentos $^{1}$ & VFCNF & KdCNF & VFCHOF & KdCHOF & Latência \\
\hline Silagem de capim napier & 41,53 & 0,0410 & 41,33 & 0,0174 & 3,35 \\
Milho & 26,69 & 0,1145 & 127,75 & 0,0243 & 8,57 \\
Farelo de soja & 39,01 & 0,1176 & 53,30 & 0,023 & 4,88 \\
Resíduo feijão & 79,26 & 0,0514 & 60,87 & 0,0177 & 4,56 \\
Torta de algodão & 22,65 & 0,1363 & 27,89 & 0,0217 & 4,57 \\
\hline
\end{tabular}

VFCNF (mL/g MS): volume final de gás referente à fração carboidratos não-fibrosos; $K d C N F\left(\% \mathrm{~h}^{-1}\right)$ : taxa de degradação referente à fração carboidratos não-fibrosos; VFCHOF (mL/g MS): volume final de gás referente à fração carboidratos fibrosos; $\mathrm{KdCHOF}\left(\% \mathrm{~h}^{-1}\right)$ : taxa de degradação referente à fração carboidratos fibrosos; Latência: horas. 


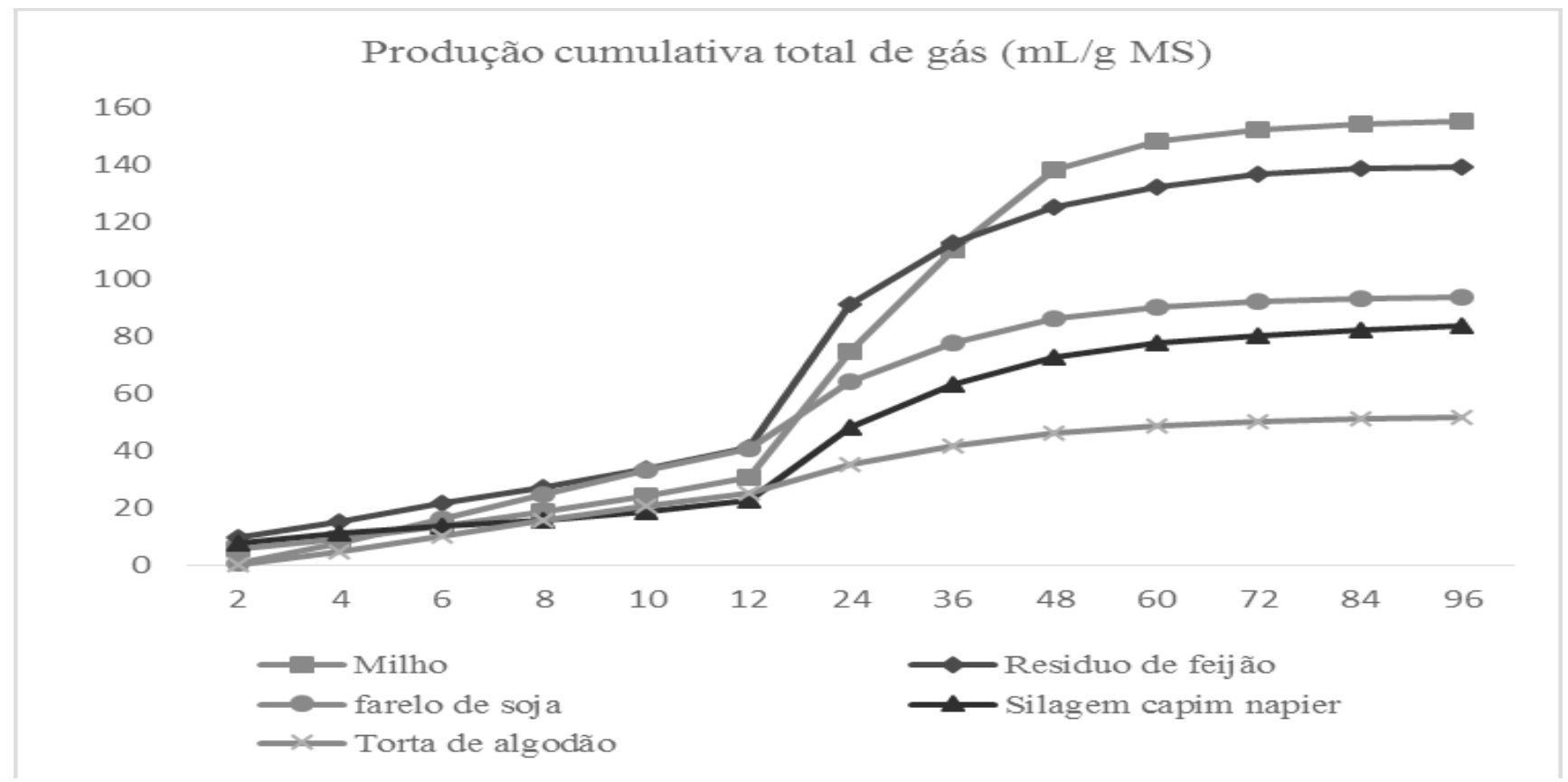

Figura 1. Produção cumulativa de gases (mL/g MS) de alimentos utilizados na composição das dietas (Cumulative gas production ( $\mathrm{mL} / \mathrm{g} \mathrm{MS}$ ) food used in the composition of diets).

no volume de gases, uma vez que, houve aumento de $8,8 \%$ no teor de CNF da dieta $0 \%$ para dieta $100 \%$ de substituição da torta de algodão por resíduo de feijão. Silva et al. (2015) trabalhando com inclusão no concentrado de torta de girassol (0, 100, 200 e $300 \mathrm{~g} / \mathrm{kg}$ de MS) também não encontrou efeito significativo para produção de gases, justificado pela baixa proporção de frações solúveis.

A taxa de degradação da fração CNF (KdCNF) apresentou aumento linear $(\mathrm{p}<0,05)$ à medida que incluiu resíduo de feijão nas dietas em substituição à torta de algodão, sendo que para cada $1 \%$ de substi- tuição ocorreu aumento de $0,0003 \% / \mathrm{h}^{-1}$. Ferro (2014) obteve aumento linear $(\mathrm{p}<0,05)$ na digestibilidade dos CNF com a inclusão do resíduo de feijão substituindo a torta de algodão em dietas para ovinos confinados, justificando o comportamento da taxa de degradação do presente trabalho.

O volume de gases da fração CHOF (VFCHOF) apresentou aumento linear significativo $(p<0,05)$ com aumento dos níveis de resíduo de feijão em substituição a torta de algodão, sendo que para cada $1 \%$ de substituição ocorreu aumento de $0,23 \mathrm{~mL} / \mathrm{g}$ de MS (tabela IV).

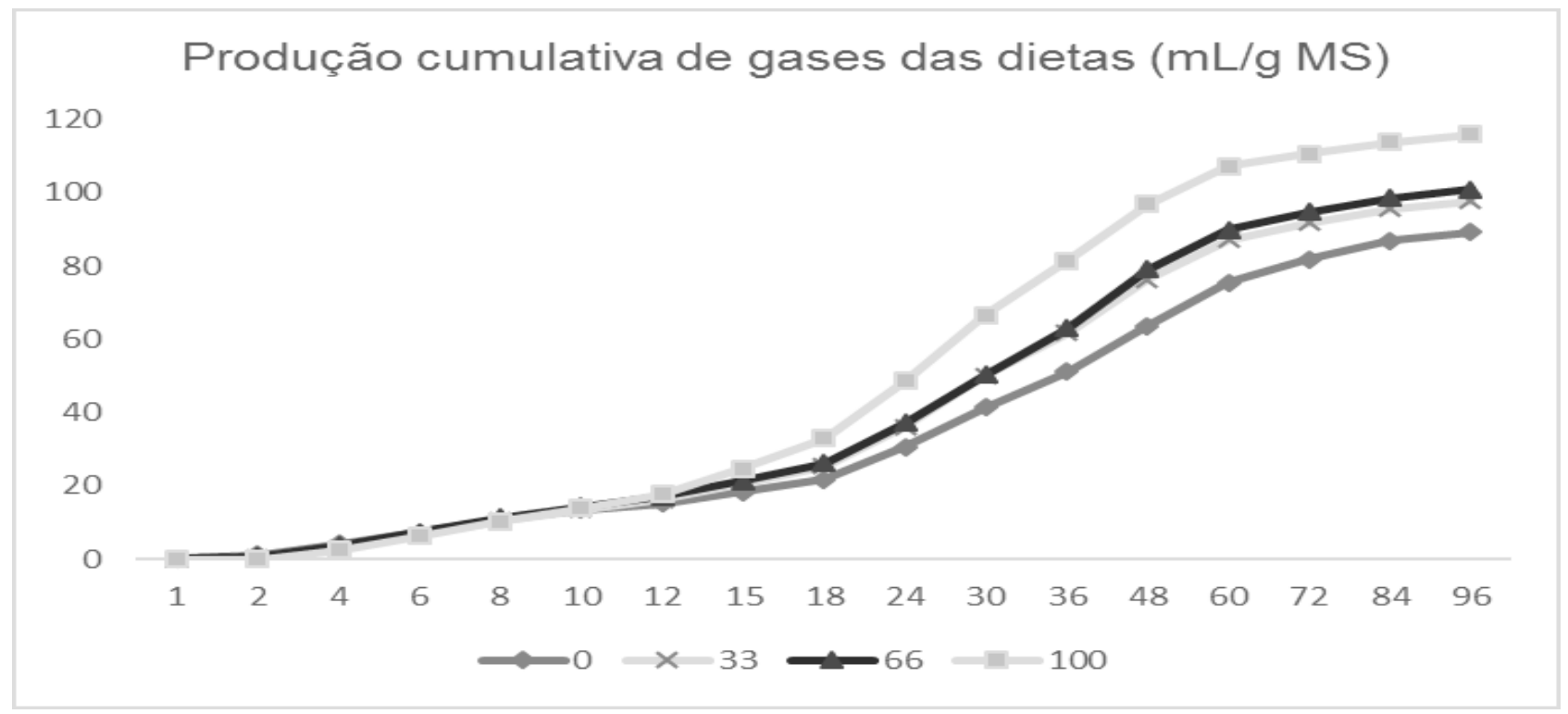

Figura 2. Produção cumulativa de gases (mL/g MS) das dietas com substituição de 0, 33, 66 e $100 \%$ do resíduo de feijão em substituição à torta de algodão (Cumulative gas production (mL/g DM) diets with replacement of $0,33,66$ and $100 \%$ of the bean residue as a substitute for cotton cake). 
Tabela IV. Parâmetros cinéticos de fermentaçao ruminal in vitro pela técnica cumulativa de gases das dietas (Kinetic parameters of in vitro ruminal fermentation by cumulative technique of diets gases).

\begin{tabular}{|c|c|c|c|c|c|c|c|}
\hline \multirow{2}{*}{ Parâmetros ${ }^{1}$} & \multicolumn{4}{|c|}{ Níveis de substituição por resíduo de feijão (\%) } & \multirow{2}{*}{ EPM } & \multirow{2}{*}{ Equação } & \multirow{2}{*}{$\mathrm{R}^{2}$} \\
\hline & 0 & 33 & 66 & 100 & & & \\
\hline VFCNF & 16,92 & 21,25 & 11,10 & 19,72 & 2,81 & $Y=17,24$ & - \\
\hline KdCNF & 0,0582 & 0,0592 & 0,0744 & 0,0924 & 0,003 & $Y=0,0534+0,0003 R F$ & 0,83 \\
\hline VFCHOF & 71,96 & 73,31 & 84,77 & 90,72 & 2,73 & $Y=70,07+0,2034 R F$ & 0,82 \\
\hline $\mathrm{KdCHOF}$ & 0,0172 & 0,0203 & 0,0224 & 0,0253 & 0,00 & $Y=0,0174+0,00007 R F$ & 0,94 \\
\hline VFtotal & 88,89 & 94,56 & 95,88 & 110,45 & 2,72 & $87,56+0,1986 \mathrm{RF}$ & 0,77 \\
\hline Latência & 9,40 & 10,05 & 10,21 & 10,30 & 0,45 & $Y=9,56+0,0085 R F$ & 0,30 \\
\hline
\end{tabular}

VFCNF (mL/g MS): volume final de gás referente à fração carboidratos não-fibrosos; $\operatorname{KdCNF~}\left(\% \mathrm{~h}^{-1}\right)$ : taxa de degradação referente à fração carboidratos não-fibrosos; VFCHOF (mL/g MS): volume final de gás referente à fração carboidratos fibrosos; $\mathrm{KdCHOF}\left(\% \mathrm{~h}^{-1}\right)$ : taxa de degradação referente à fração carboidratos fibrosos; VFtotal: volume final total de gás (ml/g MS); Latência: horas; horas; $R^{2}$ : Coeficiente determinativo; EPM: erro-padrão da média.

A taxa de degradação da fração $\mathrm{CHOF}(\mathrm{KdCHOF})$ apresentou comportamento linear $(\mathrm{p}<0,05)$ com o aumento de $0,00007 \mathrm{~mL} / \mathrm{g}$ de MS conforme substituiu a torta de algodão por resíduo de feijão (tabela IV). A melhoria na taxa de degradação pode ter sido influenciada pela redução em 3,9\% da fração FDNcp das dietas, associada com o aumento da fração CNF que pode ter potencializado a digestão da fibra das dietas (tabela II). Silva et al. (2015) obtiveram efeitos contrários ao presente trabalho quando incluíram torta de girassol $(0,100,200$ e $300 \mathrm{~g} / \mathrm{kg}$ de MS) que reduziu a taxa de degradação da fração de lenta digestão, justificado pela composição química do alimento, uma vez que a taxa de degradação pode influenciar na taxa de repleção ruminal, levando a uma limitação do consumo de MS, consequentemente prejudicando a produção animal.

$\mathrm{O}$ volume final total de gases apresentou aumento linear significativo $(p<0,05)$ de $0,19 \mathrm{~mL} / \mathrm{g}$ de MS à medida que aumentou a inclusão do resíduo de feijão em substituição à torta de algodão nas dietas (tabela IV).

Getachew et al. (2004) observaram alta correlação entre a extensão de produção de gases e a composição química dos alimentos. Os gases produzidos durante o processo fermentativo são principalmente o $\mathrm{CO}_{2}$ e o $\mathrm{CH}_{4}$, e a sua formação é resultado principalmente da fermentação dos carboidratos dos alimentos à AGCC (Jenkins, 1993; Cone e Van Gelder, 1999). A contribuição da fermentação das proteínas e dos lipídeos para a produção desses gases é muito pequena, sendo no caso dos lipídeos praticamente inexpressivos, apesar de serem potencialmente digestíveis e colaborarem para a digestibilidade total da matéria seca.
A latência apresentou aumento linear significativo $(p<0,05)$, aumentando 0,008 horas a cada $1 \%$ de substituição da torta de algodão por resíduo de feijão (tabela IV). Mertens (1997), afirmou que o tempo de colonização (latência) é um parâmetro importante, pois está relacionado com a degradação da fração fibrosa. Segundo Azevêdo et al. (2003) o tempo de colonização é proporcional à concentração de FDN, contrariando o comportamento do presente trabalho, pois conforme se incluiu o resíduo de feijão substituindo a torta de algodão reduziu o teor de FDN das dietas.

A energia metabolizável (EM) (MJ/kg MS), digestibilidade da $\mathrm{MO}$ e a quantidade de ácidos graxos de cadeia curta (AGCC) apresentaram aumento linear significativo $(\mathrm{p}<0,05)$ de 0,008 MJ/ $\mathrm{kg} \mathrm{MS} ; 0,58 \mathrm{~g} / \mathrm{kg}$ de MS e $0,0014 \mathrm{mmol} / \mathrm{g}$ MS, respectivamente, à medida que aumentou a inclusão do resíduo de feijão em substituição a torta de algodão (tabela V). Esse comportamento pode ser explicado pelo aumento das taxas de degradação da fração de alta e lenta digestão, caracterizado pelo valor nutricional do resíduo de feijão quando substituiu a torta de algodão.

\section{CONCLUSÃO}

A utilização do resíduo feijão melhorou os parâmetros fermentativos in vitro como produção de gases, taxa de degradação e latência, promovendo aumento da disponibilidade de energia metabolizável, digestibilidade da matéria orgânica e ácidos graxos de cadeia curta. Desta forma, o resíduo de feijão pode substituir

Tabela V. Estimativa do perfil de fermentação ruminal in vitro de dietas com inclusão do resíduo de feijão em substituição à torta de algodão (Profile estimate of in vitro ruminal fermentation of diets with the inclusion of bean residue replacing cotton cake).

\begin{tabular}{|c|c|c|c|c|c|c|c|}
\hline \multirow{2}{*}{ Parâmetros ${ }^{1}$} & \multicolumn{4}{|c|}{ Níveis de substituição por resíduo de feijão (\%) } & \multirow{2}{*}{ EPM } & \multirow{2}{*}{ Equação } & \multirow{2}{*}{$\mathrm{R}^{2}$} \\
\hline & 0 & 33 & 66 & 100 & & & \\
\hline EM (MJ/kg MS) & 4,04 & 4,35 & 4,39 & 5,01 & 0,05 & $Y=4,00+0,008 R F$ & 0,84 \\
\hline $\mathrm{DMO}(\mathrm{g} / \mathrm{kg})$ & 270,75 & 291,00 & 293,82 & 334,40 & 3,69 & $Y=268,49+0,58 R F$ & 0,84 \\
\hline AGCC $(\mathrm{mmol} / \mathrm{g} \mathrm{MS})$ & 0,26 & 0,31 & 0,32 & 0,42 & 0,00 & $Y=0,26+0,0014 R F$ & 0,84 \\
\hline
\end{tabular}

EM: energia metabolizável; DMO: digestibilidade da matéria orgânica; AGCC: ácidos graxos de cadeia curta; MS: Matéria seca; $\mathrm{R}^{2}$ : Coeficiente determinativo; EPM: erro-padrão da média. 
a torta de algodão $100 \%$ no concentrado observando o resultado das variáveis estudadas.

\section{BIBLIOGRAFIA}

Armentano, L. and Pereira, M. 1997. Simposium: Meeting the fiber requirements of dairy cows. Measuring the effectives of fiber by animal trial. J Dairy Sci, 80: 1416-1425.

Azevêdo, J.A.G.; Pereira, J.C.; Queiroz, A.C.; Carneiro, P.C.S.; Lana, R.P.; Barbosa, M.H.P.; Fernandes, A.M. e Rennó, F.P. 2003. Composição químico- bromatológica, fracionamento de carboidratos e cinética da degradação in vitro da fibra de três variedades de cana-de-açúcar (Saccharum spp.). Rev Bras Zootecn, 32: 1443-1453.

Azevêdo, J.A.G.; Valadares Filho, S.C.; Detmann, E.; Pina, D.S.; Pereira, L.G.R;Oliveira, K.A.M.; Fernandes, H.J. e Souza, N.K.P. 2011 . Predição de frações digestíveis e valor energético de subprodutos agrícolas e agroindustriais para bovinos. Rev Bras Zootecn, 40: 391-402.

Carvalho, G.G.P.; Pires, A.J.V.; Silva, F.F.; Veloso, C.M.; Silva, R.R.; Silva, H.G.O.; Bonomo, P. e Mendonça, S.S. 2004. Comportamento ingestivo de cabras leiteiras a limentadas com Farelo de cacau ou torta de dendê. Pesqui Agropecu Bras, 39: 919-925.

Casali, A.O.; Detmann, E.; Valadares Filho, S.C.; Pereira, J.C.; Henriques, L.T.; Freitas, S.G. e Paulino, M.F. 2008. Influência do tempo de incubação e do tamanho de partículas sobre os teores de compostos indigestíveis em alimentos e fezes bovinas obtidos por procedimentos in situ. Rev Bras Zootec, 37: 335-342.

Companhia Nacional de Abastecimento. Indicadores da Agropecuária. Cone, J.W. and Van Gelder, A.H. 1999. Influence of protein fermentation on gas production profiles. Anim Feed Sci Technol, 76: 251-264.

Detmann, E.; Souza, M.A.; Valadares Filho, S.C.; Queiroz, A.C.; Berchielli, T.T.; Saliba, E.O.S.; Cabral, L.S.; Pina, D.S.; Ladeira, M.M. e Azevêdo, J.A.G. 2012. Métodos para análises de alimentos. $1^{a} \mathrm{ed}$. Editora UFV. Viçosa. Brasil.

Ferro, M.M. 2014. Resíduo do beneficiamento do feijão em dietas para ovinos confinados. Dissertação (Mestrado em Ciência Animal). Programa de Pós-Graduação em Ciência Animal. Universidade Federal de Mato Grosso. 99 pp.

Fonseca, A.A. 2015. Resíduo de feijão na suplementação de vacas leiteiras sob pastejo. Dissertação (Mestrado em Ciência Animal). Programa de Pós-Graduação em Ciência Animal. Universidade Federal de Mato Grosso. 75 pp.

Getachew, G.; Blummel, M.; Makkar, H.P.S. and Becker, K. 2002. In vitro gas measuring techniques for assessment of nutritional quality of feeds: a review. Anim Feed Sci Technol, 72: 261-281.

Getachew, G.; Depeters, E.J. and Robinson, P.H. 2004. In vitro gas production provides effective method for assessing ruminant feeds. Calif Agricult, 58: 54-58.

Jenkins, T.C. 1993. Lipid metabolism in the rumen. J Dairy Sci, 76: 3851-3863.
Licitra, G.; Hernandez, T.M. and Van Soest, P.J. 1996. Standardization of procedures for nitrogen fractionation of ruminant feeds. Anim Feed Sci Technol, 57: 347-358.

McDougall, E.I. 1948. Studies on ruminant saliva. 1. The composition and output of sheep's saliva. Biochem J, 43: 99-109.

Magalhães, A.L.R.; Zorzi, K.; Queiroz, A.C.; Mello, R.; Detmann, E. e Pereira, J.C. 2008. Resíduo proveniente do beneficiamento do feijão (Phaseolus vulgaris L.) em rações para vacas em lactação: consumo, digestibilidade, produção e composição do leite e eficiência de alimentação. Rev Bras Zootec, 37: 529-537.

Malafaia, P.A.M.; Valadares Filho, S.C.; Vieira, R.A.M.; Silva, J.F.C. e Pereira, J.C. 1998. Cinética ruminal de alguns alimentos investigada por técnicas gravimétricas e metabólicas. Rev Bras Zootecn, 27: 370-380.

MAPA. Ministério da Agricultura, Pecuária e Abastecimento. 2002. Portaria ${ }^{\circ} 85$, de 6 de março de 2002.

Marcondes, M.I.; Valadares Filho, S.C.; Detmann, E.; Valadares, R.F.D.; Costa e Silva, L.F. e Fonseca, M.A. 2009. Degradação ruminal e digestibilidade intestinal da proteína bruta de alimentos para bovinos. Rev Bras Zootecn, 38: 2247-2257.

Mertens, D.R. 1997. Creating a system for meeting the fiber requirements of dairy cows. J Dairy Sci, 80: 1463-1481.

Pell, A.N. and Schofield, P. 1993. Computerized monitoring of gas production to measure forage digestion in vitro. J Dairy Sci, 76: 1063-1073.

Piona, M.N.M. 2010. Caroço de algodão como ingrediente na dieta de cordeiros em confinamento. Dissertação (Mestrado em Ciência Animal). Programa de Pós-Graduação em Ciência Animal. Universidade Federal de Mato Grosso. 73 pp.

Rodrigues Filho, J.A.; Camarão, A.P. e Azevedo, J.P.C. 2001. Utilização da torta de amêndoa de dendêna alimentação de ruminantes. Embrapa Amazônia Oriental, documentos 111. 24 pp.

Schofield, P.; Pitt, R.E. and Pell, A.N. 1994. Kinetics of fiber digestion from in vitro gas production. J Anim Sci, 72: 2980-2991.

Silva, L.H.X.; Goes, R.H.T.B.; Carneiro, M.M.Y.; Burin, P.C.; Oliveira, E.R.; Souza, K.A.; Ítavo, L.C.V.; Branco, A.F. e Oliveira, R.T. Produção total de gases e degradabilidade in vitro de dietas com torta de girassol. Arch Zootec, 64: 365-371.

Sniffen, C.J.; O'Connor, J.D.; Van Soest, P.J.; Fox, D.G. and Russell, J.B. 1992. A net carbohydrate and protein system for evaluating cattle diets: II. Carbohydrate and protein availability. J Anim Sci, 70: 3562-3577. 